FUNCTION SPACES XII

BANACH CENTER PUBLICATIONS, VOLUME 119

INSTITUTE OF MATHEMATICS

POLISH ACADEMY OF SCIENCES

WARSZAWA 2019

\title{
INTERPOLATION, EXTRAPOLATION, MORREY SPACES AND LOCAL ENERGY CONTROL FOR THE NAVIER-STOKES EQUATIONS
}

\author{
PIERRE GILLES LEMARIÉ-RIEUSSET \\ LaMME, Univ Evry, CNRS, Université Paris-Saclay, 91025 Évry, France \\ E-mail: pierregilles.lemarierieusset@univ-evry.fr
}

\begin{abstract}
Barker recently proved new weak-strong uniqueness results for the Navier-Stokes equations based on a criterion involving Besov spaces and a proof through interpolation between Besov-Hölder spaces and $L^{2}$. We improve slightly his results by considering Besov-Morrey spaces and interpolation between Besov-Morrey spaces and $L_{\text {uloc }}^{2}$.
\end{abstract}

1. The Navier-Stokes equations. Let $\vec{u}_{0}$ a divergence-free vector field on $\mathbb{R}^{3}$. We shall consider weak solutions to the Cauchy initial value problem for the Navier-Stokes equations which satisfy energy estimates.

The differential Navier-Stokes equations read as

$$
\begin{gathered}
\partial_{t} \vec{u}+\vec{u} \cdot \vec{\nabla} \vec{u}=\Delta \vec{u}-\vec{\nabla} p \\
\operatorname{div} \vec{u}=0 \\
\vec{u}(0, .)=\vec{u}_{0} .
\end{gathered}
$$

Under reasonable assumptions, the problem is equivalent to the following integrodifferential problem:

$$
\vec{u}=e^{t \Delta} \vec{u}_{0}-B(\vec{u}, \vec{u})(t, x)
$$

where

$$
B(\vec{u}, \vec{v})=\int_{0}^{t} e^{(t-s) \Delta} \mathbb{P} \operatorname{div}(\vec{u} \otimes \vec{v}) d s
$$

and $\mathbb{P}$ is the Leray projection operator. (See [LR 2, LR 6] for details).

2010 Mathematics Subject Classification: 35K55, 35Q30, 76D05.

Key words and phrases: Navier-Stokes equations, Morrey spaces, Besov spaces, uniformly locally square integrable functions, weak-strong uniqueness.

The paper is in final form and no version of it will be published elsewhere. 
Weak Leray solutions for the Navier-Stokes equations. When $\vec{u}_{0} \in L^{2}$, Leray proved existence of solutions $\vec{u}$ on $(0,+\infty) \times \mathbb{R}^{3}$ such that:

- $\vec{u} \in L_{t}^{\infty} L_{x}^{2} \cap L_{t}^{2} \dot{H}_{x}^{1}$

- $\lim _{t \rightarrow 0^{+}}\left\|\vec{u}(t, .)-\vec{u}_{0}\right\|_{2}=0$,

- we have the Leray energy inequality

$$
\|\vec{u}(t, .)\|_{2}^{2}+2 \int_{0}^{t}\|\vec{\nabla} \otimes \vec{u}\|_{2}^{2} d s \leq\left\|\vec{u}_{0}\right\|_{2}^{2} .
$$

Such solutions are weak solutions: the derivatives in the Navier-Stokes solutions are taken in the sense of distributions. Those solutions (that satisfy the energy inequality (2)) are called Leray weak solutions.

When $\vec{u}_{0} \in L^{2}$, Leray's proof of existence of solutions [Le] is based on mollification, energy estimates and compactness arguments:

- he solves

$$
\partial_{t} \vec{u}_{\epsilon}+\left(\varphi_{\epsilon} * \vec{u}_{\epsilon}\right) \cdot \vec{\nabla} \vec{u}_{\epsilon}=\Delta \vec{u}_{\epsilon}-\vec{\nabla} p_{\epsilon}
$$

with $\operatorname{div} \vec{u}_{\epsilon}=0$ and $\vec{u}_{\epsilon}(0,)=.\vec{u}_{0}$. Here, $\varphi \in \mathcal{D}, \int \varphi d x=1$ and $\varphi_{\epsilon}(x)=\frac{1}{\epsilon^{3}} \varphi\left(\frac{x}{\epsilon}\right)$;

- the solution holds on an interval $\left(0, T_{\epsilon}\right)$ where $T_{\epsilon}$ depends on $\epsilon$ and on $\left\|\vec{u}_{0}\right\|_{2}$ and we have the equality

$$
\left\|\vec{u}_{\epsilon}(t, .)\right\|_{2}^{2}+2 \int_{0}^{t}\left\|\vec{\nabla} \otimes \vec{u}_{\epsilon}\right\|_{2}^{2} d s=\left\|\vec{u}_{0}\right\|_{2}^{2} ;
$$

- the solution is then global; moreover by Rellich theorem, we find a subsequence that converges strongly in $\left(L_{t}^{2} L_{x}^{2}\right)_{\text {loc }}$ to a Leray solution $\vec{u}$.

Such solutions (i.e. obtained by this mollification/extraction process) will be called in the following restricted Leray weak solutions.

Restricted Leray solutions satisfy the Leray energy inequality which takes into account the energy on the whole space. But they enjoy as well a pointwise inequality property: for a non-negative locally finite measure $\mu$ we have

$$
\partial_{t}\left(|\vec{u}|^{2}\right)+2|\vec{\nabla} \otimes \vec{u}|^{2}=\Delta\left(|\vec{u}|^{2}\right)-\operatorname{div}\left(\left(2 p+|\vec{u}|^{2}\right) \vec{u}\right)-\mu .
$$

Leray solutions that enjoy the pointwise energy inequality are called suitable Leray solutions [KN].

Local weak Leray solutions. The pointwise energy inequality allows one [LR 1, LR 2] to develop a theory of weak solutions with infinite energy. Consider $\vec{u}_{0}$ a divergence-free vector field that is uniformly locally square integrable:

$$
\sup _{x_{0} \in \mathbb{R}^{3}} \int_{\left|x-x_{0}\right|<1}\left|\vec{u}_{0}(x)\right|^{2} d x<+\infty .
$$

A local Leray solution on $(0, T) \times \mathbb{R}^{3}$ is a solution such that

- $\vec{u} \in L_{t}^{\infty}\left(L_{\mathrm{uloc}}^{2}\right) \cap\left(L_{t}^{2} \dot{H}_{x}^{1}\right)_{\mathrm{uloc}}$,

- for all compact subsets $K$ of $\mathbb{R}^{3}, \lim _{t \rightarrow 0^{+}} \int_{K}\left|\vec{u}(t, .)-\vec{u}_{0}\right|^{2} d x=0$,

- we have the pointwise energy inequality (3).

Local in time existence of restricted local Leray solutions has been proved for a positive $T$ that depends only on $\left\|\vec{u}_{0}\right\|_{L_{\text {uloc }}^{2}}$ (see Section 8. 
2. The Prodi-Serrin criterion for weak-strong uniqueness. Based on a compactness criterion, the proof of existence of Leray solutions does not provide any clue on the would-be uniqueness of the solution to the Cauchy initial value problem.

A classical case of uniqueness of Leray weak solutions is Serrin's criterion for weakstrong uniqueness $\left[\mathrm{Pr}\right.$, Se. If $\vec{u}_{0} \in L^{2}$ and if the Navier-Stokes equation has a solution $\vec{u}$ on $(0, T)$ such that

$$
\vec{u} \in X_{T}=L_{t}^{p} L_{x}^{q} \text { with } \frac{2}{p}+\frac{3}{q}=1 \text { and } 2 \leq p<+\infty
$$

then if $\vec{v}$ is a Leray solution we have $\vec{u}=\vec{v}$ on $(0, T)$.

The proof of the criterion is based on the fact that if $\vec{v}$ is a Leray solution and if $\vec{u}$ is the mild solution with $\|\vec{u}\|_{X_{T}}<+\infty$, then the difference $\vec{w}=\vec{u}-\vec{v}$ satisfies a Gronwall estimate:

$$
\|\vec{w}(t, .)\|_{2}^{2}+2 \int_{0}^{t}\|\vec{\nabla} \otimes \vec{w}\|_{2}^{2} d s \leq 2 \int_{0}^{T}\left|\int \vec{u} \cdot(\vec{w} \cdot \vec{\nabla} \vec{w}) d x\right| d s .
$$

We have (for $\frac{2}{p}+\frac{3}{q}=1$ )

$$
\|\vec{u} \otimes \vec{w}\|_{2} \leq C\|\vec{u}\|_{q}\|\vec{w}\|_{2}^{2 / p}\|\vec{\nabla} \otimes \vec{w}\|_{2}^{3 / q}
$$

so that

$$
\left|\int \vec{u} \cdot(\vec{w} \cdot \vec{\nabla} \vec{w}) d x\right| \leq\|\vec{u}\|_{q}\|\vec{w}\|_{2}^{2 / p}\|\vec{\nabla} \otimes \vec{w}\|_{2}^{1+3 / q}=\|\vec{u}\|_{q}\left(\|\vec{w}\|^{2}\right)^{1 / p}\left(\|\vec{\nabla} \otimes w\|^{2}\right)^{1-1 / p} .
$$

Thus, we find

$$
2 \int_{0}^{T}\left|\int \vec{u} \cdot(\vec{w} \cdot \vec{\nabla} \vec{w}) d x\right| d s \leq C \int_{0}^{t}\|\vec{u}\|_{q}^{p}\|\vec{w}\|_{2}^{2} d s+\int_{0}^{t}\|\vec{\nabla} \otimes \vec{w}\|_{2}^{2} d s .
$$

The fact that $\|\vec{w}(0, .)\|_{2}^{2}=0$ and $\|\vec{w}(t, .)\|_{2}^{2} \leq C \int_{0}^{t}\|\vec{u}\|_{q}^{p}\|\vec{w}\|_{2}^{2} d s$ then gives $\vec{w}=0$ on $(0, T)$.

We may comment a little further in the case $2<p<+\infty$. In that case, the bilinear operator $B$ (given by (1)) is bounded on $X_{T}=L_{t}^{p} L_{x}^{q}$. Thus, we find that the existence of $T>0$ and of a solution in $L^{p} L^{q}$ with $\frac{2}{p}+\frac{3}{q}=1$ is equivalent to the existence of $T^{\prime}$ such that $e^{t \Delta} \vec{u}_{0} \in L^{p} L^{q}$ on $\left(0, T^{\prime}\right)$ (and on $(0,+\infty)$, since $\left.\vec{u}_{0} \in L^{2}\right)$. Using the thermic characterization of Besov spaces, we can see that this is equivalent with

$$
\vec{u}_{0} \in \dot{B}_{q, p}^{-1+3 / q} \text {. }
$$

Thus, the initial value is not only in $L^{2}$, but it must belong as well to a Besov space with a better regularity than provided by the embedding

$$
L^{2} \subset \dot{B}_{q, 2}^{-3 / 2+3 / q} \subset \dot{B}_{q, p}^{-3 / 2+3 / q} .
$$

3. The Koch and Tataru theorem and T. Barker's question. We may now wonder how to generalize the Prodi-Serrin criterion. It means: given $\vec{u}_{0} \in L^{2}$ and weak Leray solutions associated to $\vec{u}_{0}$, find a space $\mathbb{X}$ (as large as possible) such that if moreover $\vec{u}_{0} \in \mathbb{X}$ then we have a solution $\vec{u} \in X_{T}$ for some space $X_{T}$ of functions on $(0, T) \times \mathbb{R}^{3}$ and such that the existence of a solution in $X_{T}$ implies that any other weak Leray solution is equal to this solution $\vec{u}$ for $0<t<T$. 
The space $\mathrm{BMO}^{-1}$. First of all, we precise which kind of space $\mathbb{X}$ we are going to study. The idea is to look at an initial value which generates a solution in some uniqueness class (where uniqueness holds for small solutions). The setting where to construct such solutions is the setting of mild solutions, as introduced by Kato [Ka]: mild solutions are constructed by the Banach contraction principle.

Due to the symmetries of the equations (if $\vec{u}$ is a solution for initial value $\vec{u}_{0}$, then $\lambda \vec{u}\left(\lambda^{2} t, \lambda\left(x-x_{0}\right)\right)$ is a solution for the initial value $\left.\lambda \vec{u}_{0}\left(\lambda\left(x-x_{0}\right)\right)\right)$, we look for spaces with norms invariant through the transforms $\vec{u}_{0}(.) \mapsto \lambda \vec{u}\left(\lambda\left(.-x_{0}\right)\right)$ (for $\left.\lambda>0\right)$. Moreover, in order to be able to define

$$
B(\vec{u}, \vec{v})=\int_{0}^{t} e^{(t-s) \Delta} \mathbb{P} \operatorname{div}(\vec{u} \otimes \vec{v}) d s
$$

at least for $\vec{u}=\vec{v}=e^{t \Delta} \vec{u}_{0}$ (first step of the Picard iteration to find a fixed-point to $\left.\vec{u}=e^{t \Delta} \vec{u}_{0}-B(\vec{u}, \vec{u})\right)$, we ask that $\int_{[0,1] \times B(0,1)}\left|e^{s \Delta} \vec{u}_{0}(y)\right|^{2} d s d y<+\infty$.

Thus, we are led to introduce the space $\mathbb{X}$ of distributions $v$ such that

- $\sup _{t>0} \sqrt{t}\left\|e^{t \Delta} v\right\|_{\infty}<+\infty$

- $\sup _{0<t, x_{0} \in \mathbb{R}^{3}}\left(t^{-3 / 2} \int_{0}^{t} \int_{B\left(x_{0}, \sqrt{t}\right)}\left|e^{s \Delta} v(y)\right|^{2} d y d s\right)^{1 / 2}<+\infty$.

This space $\mathbb{X}$ has been identified by Koch and Tataru $[\overline{K o c T}$ : this is the TriebelLizorkin space $\dot{F}_{\infty, 2}^{-1}$, or equivalently the space $\mathrm{BMO}^{-1}=\sqrt{-\Delta}$ BMO. Moreover, they proved the following theorem:

TheOREM 3.1. For $0<T \leq \infty$, define

$$
\|\vec{u}\|_{X_{T}}=\sup _{0<t<T} \sqrt{t}\|\vec{u}(t, .)\|_{\infty}+\sup _{0<t<T, x_{0} \in \mathbb{R}^{3}}\left(t^{-3 / 2} \int_{0}^{t} \int_{B\left(x_{0}, \sqrt{t}\right)}|\vec{u}(s, y)|^{2} d y d s\right)^{1 / 2} .
$$

There exists $C_{0}$ (which does not depend on $T$ ) such that if $T \in(0,+\infty]$, if $\vec{u}$ and $\vec{v}$ are defined on $(0, T) \times \mathbb{R}^{3}$ and if

$$
B(\vec{u}, \vec{v})=\int_{0}^{t} e^{(t-s) \Delta} \mathbb{P} \operatorname{div}(\vec{u} \otimes \vec{v}) d s
$$

then

$$
\|B(\vec{u}, \vec{v})\|_{X_{T}} \leq C_{0}\|\vec{u}\|_{X_{T}}\|\vec{v}\|_{X_{T}} .
$$

Corollary 3.2. If $\left\|e^{t \Delta} \vec{u}_{0}\right\|_{X_{T}}<\frac{1}{4 C_{0}}$, then the integral Navier-Stokes equations have a solution on $(0, T)$ such that $\|\vec{u}\|_{X_{T}} \leq 2\left\|e^{t \Delta} \vec{u}_{0}\right\|_{X_{T}}$.

This is the unique solution such that $\|\vec{u}\|_{X_{T}} \leq \frac{1}{2 C_{0}}$.

A special case of initial data that leads to a solution in some $X_{T}$ is given by the subspace $\mathrm{VMO}^{-1}$ of $\mathrm{BMO}^{-1}$.

DEFINITION 3.3. $\mathrm{VMO}^{-1}$ is the closure of compactly supported functions in $\mathrm{BMO}^{-1}$.

If $\vec{u}_{0} \in \mathrm{VMO}^{-1}$, then $\lim _{T \rightarrow 0}\left\|e^{t \Delta} \vec{u}_{0}\right\|_{X_{T}}=0$. Remark that we have the embedding $\dot{B}_{q, p}^{-1+3 / q} \subset \mathrm{VMO}^{-1}$ for $2<p<+\infty$ and $\frac{2}{p}+\frac{3}{q}=1$. As a matter of fact, we may consider $\mathrm{VMO}^{-1}$ as a limit case for the scale of spaces $\dot{B}_{q, p}^{-1+3 / q}$. Thus, Barker [Ba] raised the following question: 
Question. If $\vec{u}_{0}$ belongs to $L^{2} \cap \mathrm{VMO}^{-1}$, does there exist a positive time $T$ such that every weak Leray solution of the Cauchy problem for the Navier-Stokes equations with $\vec{u}_{0}$ as initial value coincide with the mild solution in $X_{T}$ ?

If $\vec{u}_{0} \in L^{2} \cap \mathrm{VMO}^{-1}$ and if $\left\|e^{t \Delta} \vec{u}_{0}\right\|_{X_{T}} \leq \frac{1}{4 C_{0}}$, then if $\vec{u}$ is a restricted Leray solution of the Navier-Stokes solutions with initial value $\vec{u}_{0}$, then $\|\vec{v}\|_{X_{T}} \leq 2\left\|e^{t \Delta} \vec{u}_{0}\right\|_{X_{T}}$. In particular, we have uniqueness of restricted Leray solutions on $(0, T)$.

As a matter of fact, this proof of local uniqueness of restricted weak Leray solutions holds for a slightly more general class:

Definition 3.4. $\mathrm{BMO}_{0}^{-1}$ is the space of distributions $u_{0}$ in $\mathrm{BMO}^{-1}$ such that

$$
\lim _{T \rightarrow 0}\left\|e^{t \Delta} u_{0}\right\|_{X_{T}}=0 .
$$

In the following, we will focus on the hypothesis $\vec{u}_{0} \in L^{2} \cap \mathrm{BMO}_{0}^{-1}$ and on the issue of uniqueness for Leray solutions.

4. The limiting case. Up to now, we do not know how to prove local uniqueness of the Leray solutions when the initial value $\vec{u}_{0}$ belongs to $L^{2} \cap \mathrm{BMO}_{0}^{-1}$. What we know for sure is that the mild solution $\vec{u}$ in $X_{T}$ belongs to $L^{\infty}\left((\epsilon, T) \times \mathbb{R}^{3}\right)$ for every positive $\epsilon \in(0, T)$. Moreover, $\vec{u}$ is a weak Leray solution and for every other Leray solution $\vec{v}$ and for $\epsilon>0$, we have

$$
\partial_{t}(\vec{u} \cdot \vec{v})=\vec{u} . \partial_{t} \vec{v}+\partial_{t} \vec{u} \cdot \vec{v}
$$

which gives for $0<\epsilon<t<T$

$$
\begin{aligned}
\int \vec{u}(t, x) \cdot \vec{v}(t, x) d x= & \int \vec{u}(\epsilon, x) \cdot \vec{v}(\epsilon, x) d x \\
& -2 \int_{\epsilon}^{t} \int \vec{\nabla} \otimes \vec{u} \cdot \vec{\nabla} \otimes \vec{v} d x d s-\int_{\epsilon}^{t} \int \vec{u} \cdot(\vec{v} \cdot \vec{\nabla} \vec{v})+\vec{v} \cdot(\vec{u} \cdot \vec{\nabla} \vec{u}) d x d s
\end{aligned}
$$

From

$\int \vec{u} \cdot(\vec{v} \cdot \vec{\nabla} \vec{v})+\vec{v} \cdot(\vec{u} \cdot \vec{\nabla} \vec{u}) d x=\int \vec{u} \cdot(\vec{v} \cdot \vec{\nabla}(\vec{v}-\vec{u}))+(\vec{v}-\vec{u}) \cdot(\vec{u} \cdot \vec{\nabla} \vec{u}) d x=\int \vec{u}((\vec{v}-\vec{u}) \cdot \vec{\nabla}(\vec{v}-\vec{u})) d x$ and letting $\epsilon$ go to 0 , we get

$$
\begin{aligned}
\int \vec{u}(t, x) \cdot \vec{v}(t, x) d x=\left\|\vec{u}_{0}\right\|_{2}^{2} & -2 \int_{0}^{t} \int \vec{\nabla} \otimes \vec{u} \cdot \vec{\nabla} \otimes \vec{v} d x d s \\
& -\lim _{\epsilon \rightarrow 0} \int_{\epsilon}^{t} \int \vec{u}((\vec{v}-\vec{u}) \cdot \vec{\nabla}(\vec{v}-\vec{u})) d x d s
\end{aligned}
$$

and (letting $\vec{v}=\vec{u}$ )

$$
\|\vec{u}(t, .)\|_{2}^{2}=\left\|\vec{u}_{0}\right\|_{2}^{2}-2 \int_{0}^{t}\|\vec{\nabla} \otimes \vec{u}\|_{2}^{2} d s .
$$

Combining those two equalities with the Leray energy inequality for $\vec{v}$

$$
\|\vec{v}(t, .)\|_{2}^{2}+2 \int_{0}^{t}\|\vec{\nabla} \otimes \vec{v}\|_{2}^{2} d s \leq\left\|\vec{u}_{0}\right\|_{2}^{2}
$$


we get the following inequality for $\vec{w}=\vec{u}-\vec{v}$ :

$$
\|\vec{w}(t, .)\|_{2}^{2}+2 \int_{0}^{t}\|\vec{\nabla} \otimes \vec{w}\|_{2}^{2} d s \leq 2 \int_{0}^{T}\left|\int \vec{u} \cdot(\vec{w} \cdot \vec{\nabla} \vec{w}) d x\right| d s .
$$

As a matter of fact, the key ingredient in Prodi-Serrin's criterion is the estimation of the integral

$$
I(\vec{u}, \vec{w})=\int_{0}^{t}\left|\int \vec{u}(. \vec{w} \cdot \vec{\nabla} \vec{w}) d x\right| d s
$$

but, if $\vec{w}=\vec{v}-\vec{u}$ with $\vec{v}$ a Leray solution and $\vec{u}$ the mild solution in $X_{T}$, we do not even know whether $I(\vec{u}, \vec{w})$ is finite.

In the limiting case of Prodi and Serrin, (for $p=2$ and $q=+\infty$ ), we write

$$
\begin{aligned}
\|\vec{w}(t, .)\|_{2}^{2}+2 \int_{0}^{t}\|\vec{\nabla} \otimes \vec{w}\|_{2}^{2} d s & \leq 2 \int_{0}^{t} \int_{0}^{t}\left|\int \vec{u}(\cdot \vec{w} \cdot \vec{\nabla} \vec{w}) d x\right| d s \\
& \leq 2 \int_{0}^{t}\|\vec{u} \otimes \vec{w}\|_{2}\|\vec{\nabla} \otimes \vec{w}\|_{2} d s
\end{aligned}
$$

and get

$$
\|\vec{w}(t, .)\|_{2}^{2} \leq \int_{0}^{t}\|\vec{u}\|_{\infty}^{2}\|\vec{w}\|_{2}^{2} d s .
$$

Of course, we may conclude under the assumption that $\vec{u} \in L_{t}^{2} L_{x}^{\infty}$. Actually, we shall not be interested in measurability issues for functions with values in a non-separable space such as $L^{\infty}$ (i.e. in Bochner measurability for instance), as we are dealing with locally integrable functions for the Lebesgue measure $d t d x$ on $(0, T) \times \mathbb{R}^{3}$. Thus, for almost every $t$ the quantity $\|\vec{u}(t, .)\|_{\infty}$ will be well defined as a measurable function of $t$, and $\vec{u} \in L_{t}^{2} L_{x}^{\infty}$ will simply mean that $\int_{0}^{T}\|\vec{u}(t, .)\|_{L^{\infty}(d x)}^{2} d t<+\infty$. (See [LR 2] for details.)

If $\vec{u} \in L_{t}^{2} L_{x}^{\infty}$ on $(0, T) \times \mathbb{R}^{3}$, the Prodi-Serrin criterion proves that every weak Leray solution $\vec{v}$ on $(0, T)$ is equal to the mild solution $\vec{u}$. (This has even be extended to the case $\vec{u} \in L_{t}^{2} \mathrm{BMO}_{x}$ by Kozono and Tanyuchi [KozT] $)$. But it is not easy to translate the condition that $\vec{u} \in L_{t}^{2} L_{x}^{\infty}$ into an equivalent assumption on $\vec{u}_{0}$. The problem comes from the fact that the bilinear operator $B$ is not bounded on $L_{t}^{2} L_{x}^{\infty}$.

On the other hand, if we only assume $\vec{u}_{0} \in L^{2} \cap \mathrm{BMO}_{0}^{-1}$, we only know the inequality $\|\vec{u}(t, .)\|_{\infty} \leq\|\vec{u}\|_{X_{T}} \frac{o(1)}{\sqrt{t}}$. We find an integrability issue near $t=0$. To check that this is actually an issue, consider the following example: take $\vec{\omega}$ a divergence-free vector field in the Schwartz class such that the Fourier transform of $\vec{\omega}$ is compactly supported in the annulus $1<|\xi|<2$; define

$$
\vec{u}_{0}(x)=\sum_{j=0}^{+\infty} 2^{j} \frac{1}{\sqrt{1+j}} \vec{\omega}\left(2^{j} x\right) ;
$$

we have $\vec{u}_{0} \in L^{2}, \vec{u}_{0} \in \dot{B}_{q, p}^{-1+3 / q} \subset \dot{B}_{\infty, p}^{-1}$ (with $2<p<+\infty$ and $\frac{2}{p}+\frac{3}{q}=1$ ) but $\vec{u}_{0} \notin \dot{B}_{\infty, 2}^{-1}$; as the bilinear operator $B$ is bounded on $L_{t}^{p} L_{x}^{q} \cap L_{t}^{2} L_{x}^{\infty}$, if we assume that the mild solution $\vec{u} \in L_{t}^{p} L_{x}^{q}$ belongs to $L_{t}^{2} L_{x}^{\infty}$, we would find that $e^{t \Delta} \vec{u}_{0} \in L_{t}^{2} L_{x}^{\infty}$, and thus $\vec{u}_{0} \in \dot{B}_{\infty, 2}^{-1}$; thus, we have $\int_{0}^{T}\|\vec{u}\|_{\infty}^{2} d t=+\infty$. 
5. Barker's theorem. In this section, we shall sketch the proof of Barker [Ba], as we shall extend it in Section 7 to the case of Besov-Morrey spaces. The main idea in the recent paper of Barker is the following one: if we want to use only the inequality

$$
\|\vec{u}(t, .)\|_{\infty} \leq\|\vec{u}\|_{X_{T}} \frac{o(1)}{\sqrt{t}}
$$

to deal with the Gronwall inequality (5), we need to assume more than $\vec{w} \in L_{t}^{\infty} L_{x}^{2}$. Indeed, we have the following easy lemma:

Lemma 5.1. Let $\delta>0$. Let $A$ and $B$ be locally bounded non-negative measurable functions on $(0, T]$ such that

$$
\lim _{t \rightarrow 0} t A(t)=0 \text { and } \sup _{0<t<T} t^{-\delta} B(t)=0 .
$$

If we have moreover, for all $t \in(0, T]$,

$$
B(t) \leq \int_{0}^{t} A(s) B(s) d s
$$

then $B=0$.

Proof. We have

$$
B(t) \leq \frac{t^{\delta}}{\delta} \sup _{0<s<t} s A(s) \sup _{0<\sigma<t} \sigma^{-\delta} B(\sigma)
$$

so that $B=0$ on $\left(0, T_{0}\right]$ as long as $\sup _{0<s<T_{0}} s A(s)<\delta$. For $t>T_{0}$, we then write $B(t) \leq \sup _{T_{0}<s<T} A(s) \int_{T_{0}}^{t} B(s) d s$ and we find $B=0$.

As $\vec{w}=\vec{v}-\vec{u}=\left(\vec{v}-e^{t \Delta} \vec{u}_{0}\right)-\left(\vec{u}-e^{t \Delta} \vec{u}_{0}\right)$, the extra information on $\|\vec{w}\|_{2}$ will be provided by the following lemma:

LEMMA 5.2. Let $\vec{u}_{0}$ be a divergence-free vector field with $\vec{u}_{0} \in L^{2}$ and let $\vec{v}$ be a weak Leray solution of the Navier-Stokes equations with initial value $\vec{u}_{0}$. If moreover

$$
\vec{u}_{0} \in\left[L^{2}, B_{\infty, \infty}^{-\gamma}\right]_{\theta, \infty} \text { for some }-1<-\gamma<0 \text { and } 0<\theta<1
$$

then there exists $\delta>0$ such that

$$
\sup _{t>0} t^{-\delta}\left\|\vec{v}(t, .)-e^{t \Delta} \vec{u}_{0}\right\|_{2}<+\infty
$$

Proof. Assume $\vec{u}_{0} \in\left[L^{2}, B_{\infty, \infty}^{-\gamma}\right]_{\theta, \infty}$. For $0<\epsilon<1$, split $\vec{u}_{0}$ in $\vec{\alpha}_{\epsilon}+\vec{\beta}_{\epsilon}$ with

$$
\left\|\vec{\alpha}_{\epsilon}\right\|_{2} \leq C_{0} \epsilon^{\theta} \text { and }\left\|\vec{\beta}_{\epsilon}\right\|_{\dot{B}_{\infty}^{-\gamma}, \infty} \leq C_{0} \epsilon^{\theta-1}
$$

where $C_{0}$ does not depend on $\epsilon$ (but depends on $\vec{u}_{0}$ ).

We have a solution $\vec{U}_{\epsilon}$ for the Navier-Stokes equations with initial value $\vec{\beta}_{\epsilon}$ such that $\left\|\vec{U}_{\epsilon}(t, .)\right\|_{\infty} \leq C_{1} t^{-\gamma / 2} \epsilon^{\theta-1}$ on an interval $\left(0, T_{\epsilon}\right)$ with $T_{\epsilon}^{(1-\gamma) / 2} \epsilon^{\theta-1}=C_{2}$. Moreover $\vec{\beta}_{\epsilon}=\vec{u}_{0}-\vec{\alpha}_{\epsilon} \in L^{2}$ and we find that $\sup _{0<t<T_{\epsilon}}\left\|\vec{U}_{\epsilon}\right\|_{2} \leq C_{3}$ and that $\left\|\vec{U}_{\epsilon}-e^{t \Delta} \vec{\beta}_{\epsilon}\right\|_{2} \leq$ $C_{4} t^{(1-\gamma) / 2} \epsilon^{\theta-1}$. 
Since $\vec{U}_{\epsilon}$ is in $L_{t}^{2} L_{x}^{\infty}$ on every bounded interval, we get

$$
\begin{aligned}
\int \vec{U}_{\epsilon}(t, x) \cdot \vec{v}(t, x) & d x=\int \vec{\beta}_{\epsilon}(x) \cdot \vec{u}_{0}(x) d x \\
& -2 \int_{0}^{t} \int \vec{\nabla} \otimes \vec{U}_{\epsilon} \cdot \vec{\nabla} \otimes \vec{v} d x d s-\int_{0}^{t} \int \vec{U}_{\epsilon}\left(\left(\vec{v}-\vec{U}_{\epsilon}\right) \cdot \vec{\nabla}\left(\vec{v}-\vec{U}_{\epsilon}\right)\right) d x d s
\end{aligned}
$$

and

$$
\left\|\vec{U}_{\epsilon}(t, .)\right\|_{2}^{2}=\left\|\vec{\beta}_{\epsilon}\right\|_{2}^{2}-2 \int_{0}^{t}\left\|\vec{\nabla} \otimes \vec{U}_{\epsilon}\right\|_{2}^{2} d s .
$$

Combining those two equalities with the Leray energy inequality for $\vec{v}$

$$
\|\vec{v}(t, .)\|_{2}^{2}+2 \int_{0}^{t}\|\vec{\nabla} \otimes \vec{v}\|_{2}^{2} d s \leq\left\|\vec{u}_{0}\right\|_{2}^{2}
$$

we get the following inequality for $\vec{W}_{\epsilon}=\vec{v}-\vec{U}_{\epsilon}$ :

$$
\left\|\vec{W}_{\epsilon}(t, .)\right\|_{2}^{2}+2 \int_{0}^{t}\left\|\vec{\nabla} \otimes \vec{W}_{\epsilon}\right\|_{2}^{2} d s \leq\left\|\vec{\alpha}_{\epsilon}\right\|_{2}^{2}+2 \int_{0}^{T}\left|\int \vec{U}_{\epsilon} \cdot\left(\vec{W}_{\epsilon} \cdot \vec{\nabla} \vec{W}_{\epsilon}\right) d x\right| d s .
$$

Thus, we get

$$
\left\|\vec{W}_{\epsilon}(t, .)\right\|_{2}^{2} \leq C_{0}^{2} \epsilon^{2 \theta}+C_{1}^{2} \epsilon^{2(\theta-1)} \int_{0}^{t} s^{-\gamma}\left\|\vec{W}_{\epsilon}(s, .)\right\|_{2}^{2} d s
$$

so that

$$
\left\|\vec{W}_{\epsilon}(t, .)\right\|_{2}^{2} \leq C_{0}^{2} \epsilon^{2 \theta} \exp \left(C_{1}^{2} \epsilon^{2(\theta-1)} \frac{t^{1-\gamma}}{1-\gamma}\right) .
$$

Now, for $\tau<1$, take $\epsilon=\tau^{\mu}$ with $\frac{1-\gamma}{2}+\mu(\theta-1)>0$. We find that, for $0<t<T_{\epsilon}$ with

$$
T_{\epsilon}=C_{2}^{2 /(1-\gamma)} \epsilon^{2(1-\theta) /(1-\gamma)}=C_{2}^{2 /(1-\gamma)} \tau^{2 \mu(1-\theta) /(1-\gamma)}\left[\text { where } \mu \frac{2(1-\theta)}{1-\gamma}<1\right],
$$

we have the inequality

$$
\begin{aligned}
\left\|\vec{v}-e^{t \Delta} \vec{u}_{0}\right\|_{2} & \leq\left\|\alpha_{\epsilon}\right\|_{2}+\left\|\vec{W}_{\epsilon}\right\|_{2}+\left\|\vec{U}_{\epsilon}-e^{t \Delta} \vec{\beta}_{\epsilon}\right\|_{2} \\
& \leq C_{0} \epsilon^{\theta}\left(1+\exp \left(\frac{C_{1}^{2}}{2(1-\gamma)} \frac{t^{1-\gamma}}{T_{\epsilon}^{1-\gamma}}\right)\right)+C_{4} t^{(1-\gamma) / 2} \epsilon^{\theta-1} .
\end{aligned}
$$

If $\tau$ is small enough, we have $\tau<T_{\epsilon}$ and we find

$$
\left\|\vec{v}(\tau, .)-e^{\tau \Delta} \vec{u}_{0}\right\|_{2} \leq C_{0} \tau^{\mu \theta}\left(1+e^{C_{1}^{2} /(2(1-\gamma))}\right)+C_{4} \tau^{(1-\gamma) / 2+\mu(\theta-1)}
$$

The lemma is proved.

Barker's theorem then reads as:

THEOREM 5.3. Let $\vec{u}_{0}$ be a divergence-free vector field with $\vec{u}_{0} \in L^{2}$ and let $\vec{v}$ be a weak Leray solution of the Navier-Stokes equations with initial value $\vec{u}_{0}$. If moreover

$$
\vec{u}_{0} \in \mathrm{BMO}_{0}^{-1} \cap \dot{B}_{q, \infty}^{-s} \text { with } 3<q<+\infty \text { and }-s>-1+\frac{2}{q}
$$

then there exists $T>0$ such that if $\vec{v}$ is a Leray solution and if $\vec{u}$ is the mild solution with $\|\vec{u}\|_{X_{T}}<+\infty$, then $\vec{u}=\vec{v}$ on $(0, T)$. 
REMARK 5.4. We have the embeddings $L^{2} \subset \dot{B}_{q, 2}^{-3 / 2+3 / q} \subset \dot{B}_{q, \infty}^{-3 / 2+3 / q}$, so that the information conveyed by the hypothesis $\vec{u}_{0} \in \dot{B}_{q, \infty}^{-s}$ is interesting only for the high frequencies of $\vec{u}_{0}$. Moreover the embeddings $L^{2}=\dot{B}_{2,2}^{0}$ and $\mathrm{BMO}^{-1} \subset \dot{B}_{\infty, \infty}^{-1}$ give

$$
L^{2} \cap \mathrm{BMO}_{0}^{-1} \subset \dot{B}_{q, q}^{-1+2 / q} \subset \dot{B}_{q, \infty}^{-1+2 / q} .
$$

Thus, the information conveyed by the hypothesis $\vec{u}_{0} \in \dot{B}_{q, \infty}^{-s}$ is not contained in the assumption $\vec{u}_{0} \in L^{2} \cap \mathrm{BMO}_{0}^{-1}$. Finally, if $-s>-1+\frac{3}{q}=\frac{2}{p}$, then we have

$$
L^{2} \cap \mathrm{BMO}_{0}^{-1} \cap \dot{B}_{q, \infty}^{-s} \subset \dot{B}_{q, q}^{-1+2 / q} \cap \dot{B}_{q, \infty}^{-s} \subset \dot{B}_{q, p}^{-1+3 / q} .
$$

Thus, the theorem is interesting only in the range $-1+\frac{2}{q}<-s \leq-1+\frac{3}{q}$, which corresponds to the gap between $L^{2} \cap \mathrm{BMO}_{0}^{-1}$ (where local uniqueness is conjectured to hold) and $\dot{B}_{q, p}^{-1+3 / q}$ (for which the Prodi-Serrin criterion shows that local uniqueness holds).

A further remark is that we have the embedding $\dot{B}_{q, \infty}^{-1+3 / q} \subset \mathrm{BMO}^{-1}$, so that we have a Prodi-Serrin criterion with $\vec{u} \in L_{t}^{p} L_{x}^{q}$ (with $\frac{2}{p}+\frac{3}{q}=1$ ) replaced with

$$
\sup _{0<t<T} t^{1 / p}\|\vec{u}\|_{q}<+\infty \text { and } \lim _{t \rightarrow 0} t^{1 / p}\|\vec{u}(t, .)\|_{q}=0 .
$$

Proof.

We may now sketch Barker's proof for Theorem 5.3. The first step is the use of interpolation inequalities in order to be able to check that $\vec{u}_{0}$ fulfills the assumptions of Lemma 5.2

- Since $\vec{u}_{0} \in L^{2}=\dot{B}_{2,2}^{0}$ and $\vec{u}_{0} \in \mathrm{BMO}^{-1} \subset \dot{B}_{\infty, \infty}^{-1}$, we have $\vec{u}_{0} \in \dot{B}_{q, q}^{-1+2 / q}$.

- Since $\vec{u}_{0} \in \dot{B}_{q, q}^{-1+2 / q} \cap \dot{B}_{q, \infty}^{-s}$, we have for $-1+\frac{2}{q}<-\sigma<-s, \vec{u}_{0} \in \dot{B}_{q, 1}^{-\sigma}$.

- The Besov space $\dot{B}_{q, 1}^{-\sigma}$ is embedded in the Sobolev space $\dot{W}^{-\sigma, q}$.

- For $q<r<\infty$, we have $\dot{W}^{-\sigma, q}=\left[L^{2}, \dot{W}^{-\delta, r}\right]_{[\theta]}$ with $\frac{1}{q}=\frac{1-\theta}{2}+\frac{\theta}{r}$ and $-s=-\theta \delta$ (complex interpolation)

- Since $\dot{W}^{-\delta, r} \subset \dot{B}_{\infty, \infty}^{-1+3 / r}$, we have $\left[L^{2}, \dot{W}^{-\delta, r}\right]_{[\theta]} \subset\left[L^{2}, \dot{B}_{\infty, \infty}^{-\delta-3 / r}\right]_{\theta, \infty}$ with

$$
-\delta=-\frac{s}{\theta}=-s \frac{1 / 2-1 / r}{1 / 2-1 / q}=-\frac{(-s)}{-1+2 / q}+O(1 / r)
$$

so that $-\delta-\frac{3}{r}>-1$ for $r$ large enough.

We may now end the proof: recall that if $\vec{v}$ is a Leray solution and if $\vec{u}$ is the mild solution with $\|\vec{u}\|_{X_{T}}<+\infty$, then the difference $\vec{w}=\vec{u}-\vec{v}$ satisfies a Gronwall estimate:

$$
\|\vec{w}(t, .)\|_{2}^{2} \leq \int_{0}^{t}\|\vec{u}\|_{\infty}^{2}\|\vec{w}\|_{2}^{2} d s .
$$

By Lemma 5.2 we have $\left\|\vec{v}(t, .)-e^{t \Delta} \vec{u}_{0}\right\|_{2}=O\left(t^{\delta}\right)$ and $\left\|\vec{u}(t, .)-e^{t \Delta} \vec{u}_{0}\right\|_{2}=O\left(t^{\delta}\right)$ for some positive $\delta$. On the other hand, we know that $\|\vec{u}(t, .)\|_{\infty}=o\left(\frac{1}{\sqrt{t}}\right)$. Using Lemma 5.1 . we find that $\vec{w}=0$, and $\vec{v}=\vec{u}$. 
6. The Prodi-Serrin criterion for Besov-Morrey spaces. Morrey spaces provide a natural tool for extending the Prodi-Serrin criterion.

Definition 6.1. For $1<r \leq q<+\infty$, we define the Morrey space $\dot{M}^{r, q}$ as the space of Lebesgue measurable functions $f$ on $\mathbb{R}^{3}$ such that

$$
\sup _{R>0, x_{0} \in \mathbb{R}^{3}} R^{3 / q-3 / r}\left(\int_{B\left(x_{0}, R\right)}|f(x)|^{r} d x\right)^{1 / r}=\|f\|_{\dot{M}^{r, q}}<+\infty .
$$

Similarly, the space $\dot{M}^{1, q}$ is the space of locally finite Borelian (signed) measure $\mu$ such that

$$
\sup _{R>0, x_{0} \in \mathbb{R}^{3}} R^{3 / q-3}\left(\int_{B\left(x_{0}, R\right)} d|\mu|\right)=\|\mu\|_{\dot{M}^{1, q}}<+\infty .
$$

Remark: For absolutely continuous measures $d \mu=f d x$ with $f \in L_{\text {loc }}^{1}$, we have

$$
\|\mu\|_{\dot{M}^{1, q}}=\sup _{R>0, x_{0} \in \mathbb{R}^{3}} R^{3 / q-3}\left(\int_{B\left(x_{0}, R\right)}|f(x)| d x\right) .
$$

The key inequality in the proof of the Prodi-Serrin criterion was the inequality (for all $\left.w \in H^{1}\right)$

$$
\|u w\|_{2} \leq C\|u\|_{q}\|w\|_{2}^{2 / p}\|\vec{\nabla} w\|_{2}^{3 / q}
$$

with $\frac{2}{p}+\frac{3}{q}=1$ and $3<q<+\infty$. If we want to replace this inequality by a more general inequality

$$
\|u w\|_{2} \leq N(u)\|w\|_{2}^{2 / p}\|\vec{\nabla} w\|_{2}^{3 / q}
$$

(again with $\frac{2}{p}+\frac{3}{q}=1$ and $3<q<+\infty$ ), then we proved in LR 3] that the existence of a finite $N(u)$ is equivalent to the fact that $u \in \dot{M}^{2, q}$, and moreover that $N(u) \approx\|u\|_{\dot{M}^{2, q}}$. (Remark that this is false in the case $p=+\infty, q=3$, where $\dot{M}^{2,3}$ has to be replaced by a set of singular multipliers.)

This leads to the following easy extension of the Prodi-Serrin criterion:

THeOREM 6.2. If $\vec{u}_{0} \in L^{2}$ and if the Navier-Stokes equations has a solution $\vec{u}$ such that

$$
\vec{u} \in L_{t}^{p} \dot{M}_{x}^{2, q} \text { with } \frac{2}{p}+\frac{3}{q}=1 \text { and } 3<q<+\infty
$$

then if $\vec{v}$ is a Leray solution we have $\vec{u}=\vec{v}$ on $(0, T)$.

If $3<q<+\infty$, the existence of $T>0$ and of a solution in $L^{p} \dot{M}^{2, q}$ with $\frac{2}{p}+\frac{3}{q}$ is equivalent to the existence of $T^{\prime}$ such that $e^{t \Delta} \vec{u}_{0} \in L^{p} \dot{M}^{2 ; q}$ on $\left(0, T^{\prime}\right)$ (and on $(0,+\infty)$, since $\vec{u}_{0} \in L^{2}$ ), thus with

$$
\vec{u}_{0} \in \dot{B}_{\dot{M}^{2, q}, p}^{-1+3 / q} .
$$

This Besov-Morrey space has been introduced in 1994 by Kozono and Yamazaki [KoY]. It is easy to check that, for $2<p<+\infty$ and $\frac{2}{p}+\frac{3}{q}=1$, we have the inequality

$$
\left\|e^{t \Delta} u_{0}\right\|_{X_{T}} \leq C_{q}\left(\int_{0}^{T}\left\|e^{t \Delta} u_{0}\right\|_{\dot{M}^{2, q}}^{p} d t\right)^{1 / p}
$$

so that we have the embedding $\dot{B}_{\dot{M}^{2, q}, p}^{-1+3 / q} \subset \mathrm{BMO}_{0}^{-1}$ for $2<p<+\infty$ and $\frac{2}{p}+\frac{3}{q}=1$. 
7. Barker's theorem and Besov-Morrey spaces. We shall extend Barker's theorem. MAIN TheOREM 7.1. If

- $\vec{u}_{0} \in L^{2} \cap \mathrm{BMO}_{0}^{-1}$

- $3<q<+\infty,-s>-1+\frac{2}{q}$ and $\vec{u}_{0} \in \dot{B}_{\dot{M}^{1, q}, \infty}^{-s}$

then there exists $T>0$ such that if $\vec{v}$ is a suitable Leray solution and if $\vec{u}$ is the mild solution with $\|\vec{u}\|_{X_{T}}<+\infty$, then $\vec{u}=\vec{v}$ on $(0, T)$.

Proof. As we shall see, the proof is very similar to Barker's proof for Theorem 5.3 [Ba]. However, we shall meet some technical issues.

We sketch the proof:

- $\vec{u}_{0} \in L^{2}=\dot{B}_{2,2}^{0}$ and $\vec{u}_{0} \in \mathrm{BMO}^{-1} \subset \dot{B}_{\infty, \infty}^{-1}$, thus $\vec{u}_{0} \in \dot{B}_{q, q}^{-1+2 / q}$.

- $\vec{u}_{0} \in \dot{B}_{q, q}^{-1+2 / q} \cap \dot{B}_{\dot{M}^{1, q}, \infty}^{-s}$ thus $\vec{u}_{0} \in \dot{B}_{\dot{M}^{p, q}, \infty}^{-\sigma}$ for $1<p<q, \frac{1}{p}=1-\theta+\frac{\theta}{q}$ and $-\sigma=-s(1-\theta)+\theta\left(-1+\frac{2}{q}\right)>-1+\frac{2}{q}$. We shall take $p>2$.

- As $p<q$, we have $\dot{B}_{q, q}^{-1+2 / q} \subset \dot{B}_{q, p}^{1+2 / q}$. Thus, for $-1+\frac{2}{q}<-\gamma<-\sigma$,

$$
\dot{B}_{q, q}^{-1+2 / q} \cap \dot{B}_{\dot{M}^{p, q}, \infty}^{-\sigma} \subset \dot{B}_{\dot{M}^{p, q}, 1}^{-\gamma} \subset \dot{W}^{-\gamma, \dot{M}^{p, q}} .
$$

We now encounter our first problem. We can no longer write $W^{-\gamma, \dot{M}^{p, q}}$ as a subspace of an interpolation space between $L^{2}$ and $\dot{B}_{\infty, \infty}^{-1+\delta}$. More precisely, let us assume $\dot{B}_{\dot{M}^{p, q}, 1}^{-\gamma} \subset$ $\left[L^{2}, \dot{B}_{\infty, \infty}^{-1+\delta}\right]_{\theta, \infty}$; by homogeneity of the norms, we must have $-\gamma-\frac{3}{q}=-(1-\theta) \frac{3}{2}-\theta(1-\delta)$. We have

$$
\left[L^{2}, \dot{B}_{\infty, \infty}^{-1+\delta}\right]_{\theta, \infty} \subset \dot{B}_{L^{r, \infty}, \infty}^{-\theta(1-\delta)}
$$

with $r=\frac{2}{1-\theta}$. In particular, for $u \in\left[L^{2}, \dot{B}_{\infty, \infty}^{-1+\delta}\right]_{\theta, \infty}$, we see that $e^{\Delta} e^{t \partial_{3}^{2}} u$ goes to 0 in $\mathcal{S}^{\prime}$ when $t$ goes to $+\infty$. But if $3 p \leq 2 q$, if $u$ depends only on $\left(x_{1}, x_{2}\right)$ and not on $x_{3}$, and if $u \in \dot{B}_{\dot{M}^{p, 2 q / 3}, 1}^{-\gamma}\left(\mathbb{R}^{2}\right)$, then $u \in \dot{B}_{\dot{M}^{p, q}, 1}^{-\gamma}\left(\mathbb{R}^{3}\right)$ and $e^{\Delta} e^{t \partial_{3}^{2}} u=e^{\Delta} u$. Thus, we have a contradiction.

We better use complex interpolation and write that

$$
W^{-\gamma, \dot{M}^{p, q}}=\left[\dot{M}^{2,2 q / p}, \dot{W}^{-\rho, \dot{M}^{r, r q / p}}\right]^{[\theta]}
$$

for $r>p, \frac{1-\theta}{2}+\frac{\theta}{r}=\frac{1}{p}, \gamma=\theta \rho$. (For interpolation of Morrey spaces, see [LR 4, LR 5].)

Then, we remark that $\dot{M}^{2,2 q / p} \subset L_{\text {uloc }}^{2}$ and write that

$$
\left[\dot{M}^{2,2 q / p}, \dot{W}^{-\rho, \dot{M}^{r, r q / p}}\right]^{[\theta]} \subset\left[\dot{M}^{2,2 q / p}, \dot{W}^{-\rho, \dot{M}^{r, r q / p}}\right]_{\theta, \infty} \subset\left[L_{\mathrm{uloc}}^{2}, \dot{B}_{\infty, \infty}^{-\rho-3 p /(r q)}\right]_{\theta, \infty} .
$$

In order to finish the proof, we thus need to use the machinery of energy control for suitable local Leray solutions [LR 2, LR 6]. This will be done in the following sections, and we shall finish the proof in Section 10

8. Weak local Leray solutions. We recall basic results for local weak Leray solutions. We endow $L_{\text {uloc }}^{2}$ with the norm

$$
\|u\|_{L_{\mathrm{uloc}}^{2}}=\sup _{k \in \mathbb{Z}^{3}}\left\|u \varphi_{0}(x-k)\right\|_{2}
$$

where $\varphi_{0}$ is a non-negative function in $\mathcal{D}$, supported in a ball $B\left(0, R_{0}\right)$ and such that $\sum_{k \in \mathbb{Z}^{3}} \varphi_{0}(x-k)=1$. 
When $\vec{u}_{0} \in L_{\text {uloc }}^{2}$, proof of existence of solutions for the Navier-Stokes equations is based on mollification, energy estimates and compactness arguments (for details, see [LR 6], Section 14.1):

- We solve

$$
\partial_{t} \vec{u}_{\epsilon}+\left(\varphi_{\epsilon} * \vec{u}_{\epsilon}\right) \cdot \vec{\nabla} \vec{u}_{\epsilon}=\Delta \vec{u}_{\epsilon}-\vec{\nabla} p_{\epsilon}
$$

with $\operatorname{div} \vec{u}_{\epsilon}=0$ and $\vec{u}_{\epsilon}(0,)=.\vec{u}_{0}$. Here, $\varphi \in \mathcal{D}, \int \varphi d x=1$ and $\varphi_{\epsilon}(x)=\frac{1}{\epsilon^{3}} \varphi\left(\frac{x}{\epsilon}\right)$. Here $\vec{\nabla} p_{\epsilon}$ is given by the Leray projection:

$$
\vec{\nabla} p_{\epsilon}=-\left(\varphi_{\epsilon} * \vec{u}_{\epsilon}\right) \cdot \vec{\nabla} u_{\epsilon}+\mathbb{P} \operatorname{div}\left(\left(\varphi_{\epsilon} * \vec{u}_{\epsilon}\right) \otimes \vec{u}_{\epsilon}\right) .
$$

- The solution holds at least on an interval $\left(0, T_{\epsilon}\right)$ where $T_{\epsilon}$ depends on $\epsilon$ and on $\left\|\vec{u}_{0}\right\|_{L_{\text {uloc }}^{2}}\left(T_{\epsilon}=\min \left(1, C_{0} \frac{\epsilon^{3 / 2}}{\left\|\vec{u}_{0}\right\|_{L_{\text {uloc }}^{2}}^{2}}\right)\right)$. Moreover, we have the inequalities, for $k \in \mathbb{Z}^{3}$,

$$
\begin{aligned}
& \int \varphi_{0}(x-k)\left|\vec{u}_{\epsilon}(t, x)\right|^{2} x+2 \int_{0}^{t} \int \varphi_{0}(x-k)\left|\vec{\nabla} \otimes \vec{u}_{\epsilon}(s, x)\right|^{2} d x d s \\
& \leq \int \varphi_{0}(x-k)\left|\vec{u}_{0}(t, x)\right|^{2} d x+C_{1} \int_{0}^{t} \int_{|x-k| \leq R_{0}}\left|\vec{u}_{\epsilon}(s, x)\right|^{2} d x d s \\
& \quad+C_{2} \int_{0}^{t} \int_{|x-k|>5 R_{0}} \frac{1}{|x-k|^{4}}\left|\vec{u}_{\epsilon}(s, x)\right|^{3} d x d s+C_{2} \int_{0}^{t} \int_{|x-k|<5 R_{0}}\left|\vec{u}_{\epsilon}(s, x)\right|^{3} d x d s .
\end{aligned}
$$

- Defining

$$
\alpha_{\epsilon}(t)=\left\|\vec{u}_{\epsilon}(t, .)\right\|_{L_{\text {uloc }}^{2}}
$$

and

$$
\beta_{\epsilon}(t)=\sup _{k \in \mathbb{Z}^{3}}\left(\int_{0}^{t} \int \varphi_{0}(x-k)\left|\vec{\nabla} \otimes \vec{u}_{\epsilon}(s, x)\right|^{2} d x d s\right)^{1 / 2},
$$

we get the inequality

$$
\begin{aligned}
\int \varphi_{0}(x-k)\left|\vec{u}_{\epsilon}(t, x)\right|^{2} d x+ & \int_{0}^{t} \int \varphi_{0}(x-k)\left|\vec{\nabla} \otimes \vec{u}_{\epsilon}(s, x)\right|^{2} d x d s \\
& \leq \alpha_{\epsilon}(0)^{2}+\frac{1}{2} \beta_{\epsilon}(t)^{2}+C_{3} \int_{0}^{t} \alpha_{\epsilon}(s)^{2} d s+C_{3} \int_{0}^{t} \alpha_{\epsilon}(s)^{6} d s
\end{aligned}
$$

so that

$$
\beta_{\epsilon}(t)^{2} \leq 2 \alpha_{\epsilon}(0)^{2}+2 C_{3} \int_{0}^{t} \alpha_{\epsilon}(s)^{2} d s+2 C_{3} \int_{0}^{t} \alpha_{\epsilon}(s)^{6} d s
$$

and finally

$$
\alpha_{\epsilon}(t)^{2} \leq 2 \alpha_{\epsilon}(0)^{2}+2 C_{3} \int_{0}^{t} \alpha_{\epsilon}(s)^{2} d s+2 C_{3} \int_{0}^{t} \alpha_{\epsilon}(s)^{6} d s .
$$

Thus, as long as $8 C_{3} t<1$ and $128 C_{3} t\left\|\vec{u}_{0}\right\|_{L_{\text {uloc }}^{2}}^{4}<1$, we find that $\alpha_{\epsilon}(t) \leq 2\left\|\vec{u}_{0}\right\|_{L_{\text {uloc }}^{2}}$ and $\beta_{\epsilon}(t) \leq 2\left\|\vec{u}_{0}\right\|_{L_{\text {uloc }}^{2}}$.

- The solution is then defined on $\left(0, \min \left(\frac{1}{8 C_{3}}, \frac{1}{128 C_{3}\left\|\vec{u}_{0}\right\|_{L_{\text {loc }}^{2}}^{4}}\right)\right)$ and controlled independently from $\epsilon$. By Rellich theorem, we find a subsequence that converges strongly in $\left(L_{t}^{2} L_{x}^{2}\right)_{\text {loc }}$ to a suitable local Leray solution $\vec{u}$. 
An important point is: assume moreover that $\vec{u}_{0} \in \mathrm{BMO}_{0}^{-1}$ and that $\left\|e^{t \Delta} \vec{u}_{0}\right\|_{X_{T}}<\frac{1}{4 C_{0}}$ (where $C_{0}$ is the constant of Theorem 3.1) then $\vec{u}_{\epsilon}$ is defined at least on $(0, T)$ and $\left\|\vec{u}_{\epsilon}\right\|_{X_{T}} \leq 2\left\|e^{t \Delta} \vec{u}_{0}\right\|_{X_{T}}$. As $T$ does not depend on $\epsilon$, we see that the local Leray solution $\vec{u}$ satisfies $\vec{u} \in X_{S}$ with $S=\min \left(T, \frac{1}{8 C_{3}}, \frac{1}{128 C_{3}\left\|\vec{u}_{0}\right\|_{L_{\text {loc }}^{2}}^{4}}\right)$ and $\|\vec{u}\|_{X_{S}} \leq 2\left\|e^{t \Delta} \vec{u}_{0}\right\|_{X_{T}}$.

Similarly, if $\vec{u}_{0} \in \dot{B}_{\infty, \infty}^{-\gamma}$ with $-1<-\gamma<0$, then $\vec{u}_{\epsilon}$ is defined at least on $(0, T)$ where $T=C\left\|\vec{u}_{0}\right\|_{\dot{B}_{\infty, \infty}^{-\gamma}}^{2 /(1-\gamma)}$ and $\sup _{0<t<T} t^{\gamma / 2}\|\vec{u}(t, .)\|_{\infty} \leq 2 \sup _{0<t<T} t^{\gamma / 2}\left\|e^{t \Delta} \vec{u}_{0}\right\|_{\infty}$. As $T$ does not depend on $\epsilon$, we see that the local Leray solution $\vec{u}$ satisfies the inequality $\sup _{0<t<S} t^{\gamma / 2}\|\vec{u}(t, .)\|_{\infty}<+\infty$ where $S=\min \left(T, \frac{1}{8 C_{3}}, \frac{1}{128 C_{3}\left\|\vec{u}_{0}\right\|_{L_{\text {loc }}^{2}}^{4}}\right)$.

9. Comparison of local weak Leray solutions. If $\vec{u}$ and $\vec{v}$ are two local weak Leray solutions, on $(0, T)$ with initial values $\vec{u}_{0}$ and $\vec{v}_{0}$, we would like to be able to estimate $\vec{u}(t,)-.\vec{v}(t,$.$) from the estimation of \vec{u}_{0}-\vec{v}_{0}$. This can be done only when at least one of the solutions is regular enough. We shall assume that $\vec{u} \in L_{t}^{2} L_{x}^{\infty}$. We sketch the computations described in [LR 6], Section 14.4.

Define $\vec{w}=\vec{u}-\vec{v}$

$$
\alpha(t)=\|\vec{w}(t, .)\|_{L_{\mathrm{uloc}}^{2}}, \quad \beta(t)=\sup _{k \in \mathbb{Z}^{3}}\left(\int_{0}^{t} \int \varphi_{0}(x-k)|\vec{\nabla} \otimes \vec{w}(s, x)|^{2} d x d s\right)^{1 / 2} .
$$

Using the suitability of $\vec{v}$ and the regularity of $\vec{v}$, we find (for $0<t<\min (1, T)$ )

$$
\begin{aligned}
& \int \varphi_{0}(x-k)|\vec{w}(t, x)|^{2} d x+\int_{0}^{t} \int \varphi_{0}(x-k)|\vec{\nabla} \otimes \vec{w}(s, x)|^{2} d x d s \\
& \leq \alpha(0)^{2}+\frac{1}{2} \beta(t)^{2}+C_{1} \int_{0}^{t} \alpha(s)^{2} d s+C_{2} \int_{0}^{t} \alpha(s)^{6} d s+C_{3} \int_{0}^{t}\|\vec{u}(s, .)\|_{\infty}^{2} \alpha(s)^{2} d s
\end{aligned}
$$

where the constants $C_{i}$ do not depend on $T, \vec{u}$, nor on $\vec{v}$. Finally, we find

$$
\begin{aligned}
\alpha(t)^{2} \leq 2 \alpha(0)^{2}+2\left(C_{1}+C_{3}\left(\|\vec{u}\|_{L_{t}^{\infty} L_{\mathrm{uloc}}^{2}}+\|\vec{v}\|_{L_{t}^{\infty} L_{\mathrm{uloc}}^{2}}\right)^{4}\right) \int_{0}^{t} \alpha(s)^{2} d s & \\
& +2 C_{3} \int_{0}^{t}\|\vec{u}(s, .)\|_{\infty}^{2} \alpha(s)^{2} d s .
\end{aligned}
$$

We have the same estimate even if $\vec{u}$ is not integrable near $t=0$. Let us only assume that $\vec{u} \in L_{t}^{2} L_{x}^{\infty}$ on every $(\epsilon, T)$ with $\epsilon>0$. Considering a time $t_{0}>0$ which is a Lebesgue point for the functions $t \mapsto \int \varphi(x-k)|\vec{u}(t, x)|^{2} d x$ and $t \mapsto \int \varphi(x-k)|\vec{u}(t, x)|^{2} d x$, we deduce that $\vec{u}$ and $\vec{v}$ are local weak Leray solutions on $\left(t_{0}, T\right)$ with initial values $\vec{u}\left(t_{0},.\right)$ and $\vec{v}\left(t_{0},.\right)$. Thus, we shall find that, for $t>t_{0}$,

$$
\begin{aligned}
& \int \varphi_{0}(x-k)|\vec{w}(t, x)|^{2} d x \leq 2 \int \varphi_{0}(x-k)\left|\vec{w}\left(t_{0}, x\right)\right|^{2} d x \\
+ & 2\left(C_{1}+C_{3}\left(\|\vec{u}\|_{L_{t}^{\infty} L_{\mathrm{uloc}}^{2}}+\|\vec{v}\|_{L_{t}^{\infty} L_{\mathrm{uloc}}^{2}}\right)^{4}\right) \int_{t_{0}}^{t} \alpha(s)^{2} d s+2 C_{3} \int_{t_{0}}^{t}\|\vec{u}(s, .)\|_{\infty}^{2} \alpha(s)^{2} d s .
\end{aligned}
$$

It is then enough to let $t_{0}$ go to 0 and then take the supremum with respect to $k$. 
10. Proof of Theorem 7.1. We may now finish the proof. We consider two solutions of the Navier-Stokes equations with initial value $\vec{u}_{0} \in L^{2} \cap \mathrm{BMO}_{0}^{-1} \cap \dot{B}_{\dot{M}^{1, q}, \infty}^{-s}$ with $-s>-1+\frac{2}{q}$ (and $3<q<+\infty$ ): we assume that $\vec{v}$ is a suitable Leray solution and $\vec{u}$ is the mild solution in $X_{T}$.

As $\vec{v}$ is suitable, $\vec{v}$ is a local Leray solution as well and we may estimate the $L_{\text {uloc }}^{2}$ norm of $\vec{u}-\vec{w}$ : defining $B(t)=\|\vec{u}(t, .)-\vec{v}(t, .)\|_{L_{\text {uloc }}^{2}}^{2}$ and

$$
A(t)=2\left(C_{1}+C_{3}\left(\|\vec{u}\|_{L_{t}^{\infty} L_{\mathrm{uloc}}^{2}}+\|\vec{v}\|_{L_{t}^{\infty} L_{\mathrm{uloc}}^{2}}\right)^{4}\right)+2 C_{3}\|\vec{u}(t, .)\|_{\infty}^{2},
$$

we get

$$
B(t) \leq \int_{0}^{t} A(s) B(s) d s .
$$

As $\lim _{t \rightarrow 0} t A(t)=0$, we shall try to apply Lemma 5.1. Thus, we shall use interpolation estimates to search for a control of $B(t)$ as $O\left(t^{-\delta}\right)$, in the spirit of Lemma 5.2

Recall that we have introduced the following numbers:

- $p$ such that $2<p<\frac{2 q}{3}$

- $1-\theta$ the barycentric coordinate of $\frac{1}{p}$ in $\left[\frac{1}{q}, 1\right]: \frac{1}{p}=(1-\theta)+\theta \frac{1}{q}$

- $-\sigma$ the corresponding point in $\left[-1+\frac{2}{q},-s\right]:-\sigma=(1-\theta)(-s)+\theta\left(-1+\frac{2}{q}\right)$

- $-\gamma$ such that $-1+\frac{2}{q}<-\gamma<-\sigma$

- $r$ such that $p<r<+\infty$

- $1-\eta$ the barycentric coordinate of $\frac{1}{p}$ in the segment $\left[\frac{1}{r}, \frac{1}{2}\right]: \frac{1}{p}=\frac{1-\eta}{2}+\frac{\eta}{r}$

- $-\rho$ the corresponding point in $[-\gamma, 0]:-\rho=\eta(-\gamma)$.

We have the following embeddings:

- $B_{2,2}^{0} \cap \mathrm{BMO}^{-1} \subset \dot{B}_{q, q}^{-1+2 / q}$

- $\dot{B}_{q, q}^{-1+2 / q} \cap \dot{B}_{\dot{M}^{1, q}, \infty}^{-s} \subset \dot{B}_{\dot{M}^{p, q}, \infty}^{-\sigma}$

- $\dot{B}_{q, q}^{-1+2 / q} \cap \dot{B}_{\dot{M}^{p, q}, \infty}^{-\sigma} \subset \dot{B}_{\dot{M}^{p, q}, 1}^{-\gamma} \subset \dot{W}^{-\gamma, \dot{M}^{p, q}}$

- $W^{-\gamma, \dot{M}^{p, q}}=\left[\dot{M}^{2,2 q / p}, \dot{W}^{-\rho, \dot{M}^{r, r q / p}}\right]^{[\eta]}$

- $\left[\dot{M}^{2,2 q / p}, \dot{W}^{-\rho, \dot{M}^{r, r q / p}}\right]^{[\eta]} \subset\left[\dot{M}^{2,2 q / p}, \dot{W}^{-\rho, \dot{M}^{r, r q / p}}\right]_{\eta, \infty} \subset\left[L_{\mathrm{uloc}}^{2}, \dot{B}_{\infty, \infty}^{-\rho-3 p /(r q)}\right]_{\eta, \infty}$.

If we take $r$ very large, we have $\eta=1-\frac{2}{p}+o(1)$ and

$$
\left.-\rho-\frac{3 p}{r q}=\left(1-\frac{2}{p}\right)(-\gamma)+o(1) \in\right]-1,0[.
$$

Thus far, we have seen that $\vec{u}_{0} \in\left[L_{\text {uloc }}^{2}, \dot{B}_{\infty, \infty}^{-\lambda}\right]_{\eta, \infty}$ for some $\eta \in(0,1)$ and some $\lambda \in(0,1)$. We shall now estimate $\vec{v}-e^{t \Delta} \vec{u}_{0}$ when $\vec{v}$ is a weak local Leray solution. For $0<\epsilon<1$, split $\vec{u}_{0}$ in $\vec{\alpha}_{\epsilon}+\vec{\beta}_{\epsilon}$ with

$$
\left\|\vec{\alpha}_{\epsilon}\right\|_{L_{\mathrm{uloc}}^{2}} \leq C_{0} \epsilon^{\eta} \text { and }\left\|\vec{\beta}_{\epsilon}\right\|_{\dot{B}_{\infty, \infty}^{-\lambda}} \leq C_{0} \epsilon^{\eta-1}
$$

where $C_{0}$ does not depend on $\epsilon$ (but depends on $\vec{u}_{0}$ ).

As $\beta_{\epsilon}=\vec{u}_{0}-\alpha_{\epsilon}$, we have $\left\|\vec{\beta}_{\epsilon}\right\|_{L_{\text {uloc }}^{2}} \leq\left\|\vec{u}_{0}\right\|_{L_{\text {uloc }}^{2}}+C_{0}$ (an estimation which does not depend on $\epsilon$ ) and we know that we have a (restricted) weak Leray solution of the Navier-Stokes equations $\vec{U}_{\epsilon}$ with initial value $\vec{\beta}_{\epsilon}$ such that $\sup _{0<t<T_{0}}\left\|\vec{U}_{\epsilon}(t, .)\right\|_{L_{\text {uloc }}^{2}} \leq C_{1}$, where $T_{0}$ and $C_{1}$ depends only on $\left\|\vec{u}_{0}\right\|_{L_{\text {uloc }}^{2}}$ (and not on $\epsilon$ ). 
As $\beta_{\epsilon} \in \dot{B}_{\infty, \infty}^{-\lambda}$, we have as well $\left\|\vec{U}_{\epsilon}(t, .)\right\|_{\infty} \leq C_{2} t^{-\lambda / 2} \epsilon^{\eta-1}$ on an interval $\left(0, T_{\epsilon}\right)$ with $T_{\epsilon}^{(1-\lambda) / 2} \epsilon^{\eta-1}=C_{3}$. It is then easy to check that $\left\|\vec{U}_{\epsilon}-e^{t \Delta} \vec{\beta}_{\epsilon}\right\|_{L_{\text {loc }}^{2}} \leq C_{4} t^{(1-\lambda) / 2} \epsilon^{\eta-1}$.

Using our results on comparison of suitable local Leray solutions, we get the following inequality for $\vec{W}_{\epsilon}=\vec{v}-\vec{U}_{\epsilon}$ and $A_{\epsilon}(t)=\sup _{k \in \mathbb{Z}^{3}} \int \varphi_{0}(x-k)\left|\vec{W}_{\epsilon}(t, x)\right|^{2} d x$ :

$$
A_{\epsilon}(t)^{2} \leq 2\left\|\alpha_{\epsilon}\right\|_{L_{\mathrm{uloc}}^{2}}^{2}+C_{5} \int_{0}^{t} A_{\epsilon}(s)^{2} d s+C_{6} \int_{0}^{t}\|\vec{u}(s, .)\|_{\infty}^{2} A_{\epsilon}(s)^{2} d s .
$$

Thus, we get

$$
A_{\epsilon}(t) \leq C_{7} \epsilon^{2 \eta}+C_{8} \int_{0}^{t} A_{\epsilon}(s)^{2} d s+C_{9} \epsilon^{2(\eta-1)} \int_{0}^{t} s^{-\lambda} A_{\epsilon}(s)^{2} d s,
$$

so that

$$
A_{\epsilon}(t) \leq C_{7} \epsilon^{2 \eta} e^{C_{8} t} \exp \left(C_{9} \epsilon^{2(\eta-1)} \frac{t^{1-\lambda}}{1-\lambda}\right) .
$$

Now, for $\tau<1$, take $\epsilon=\tau^{\mu}$ with $\frac{1-\lambda}{2}+\mu(\eta-1)>0$. We find that, for $0<t<$ $\min \left(T_{\epsilon}, T_{0}\right)$ with

$$
T_{\epsilon}=C_{3}^{2 /(1-\lambda)} \epsilon^{2(1-\eta) /(1-\lambda)}=C_{3}^{2 /(1-\lambda)} \tau^{\mu 2(1-\eta) /(1-\lambda)} \quad\left[\text { where } \mu \frac{2(1-\eta)}{1-\lambda}<1\right],
$$

we have the inequality

$$
\begin{aligned}
\left\|\vec{v}-e^{t \Delta} \vec{u}_{0}\right\|_{L_{\text {uloc }}^{2}} & \leq\left\|\alpha_{\epsilon}\right\|_{2}+\left\|\vec{W}_{\epsilon}\right\|_{2}+\left\|\vec{U}_{\epsilon}-e^{t \Delta} \vec{\beta}_{\epsilon}\right\|_{2} \\
& \leq C_{10} \epsilon^{\eta}\left(1+e^{C_{11} t^{1-\lambda} / T_{\epsilon}^{1-\lambda}}\right)+C_{4} t^{(1-\lambda) / 2} \epsilon^{\eta-1} .
\end{aligned}
$$

If $\tau$ is small enough, we have $\tau<T_{\epsilon}$ and we find

$$
\left\|\vec{v}(\tau, .)-e^{\tau \Delta} \vec{u}_{0}\right\|_{2} \leq C_{0} \tau^{\mu \eta}\left(1+e^{C_{1}^{2} /(2(1-\lambda))}\right)+C_{4} \tau^{(1-\lambda) / 2+\mu(\eta-1)} .
$$

The theorem is proved.

\section{References}

[Ba] T. Barker, Uniqueness Results for weak Leray-Hopf solutions of the Navier-Stokes system with initial values in critical spaces, J. Math. Fluid Mech. 20 (2018), 133-160.

[CKN] L. Caffarelli, R. Kohn, L. Nirenberg, Partial regularity of suitable weak solutions of the Navier-Stokes equations, Comm. Pure Appl. Math. 35 (1982), 771-831.

[Ka] T. Kato, Nonlinear evolution equations in Banach spaces, in: 1965 Proc. Sympos. Appl. Math. 17, Amer. Math. Soc., Providence, 1965, 50-67.

[KocT] H. Koch, D. Tataru, Well-posedness for the Navier-Stokes equations, Adv. Math. 157 (2001), 22-35.

[KozT] H. Kozono, Y. Taniuchi, Bilinear estimates in BMO and the Navier-Stokes equations, Math. Z. 235 (2000), 173-194.

[KoY] H. Kozono, M. Yamazaki, Semilinear heat equations and the Navier-Stokes equations with distributions in new function spaces as initial data, Comm. Partial Differential Equations 19 (1994), 959-1014.

[LR 1] P. G. Lemarié-Rieusset, Solutions faibles d'énergie infinie pour les équations de NavierStokes dans $\mathbb{R}^{3}$, C. R. Acad. Sci. Paris Sér. I Math. 328 (1999), 1133-1138. 
[LR 2] P. G. Lemarié-Rieusset, Recent developments in the Navier-Stokes problem, Chapman \& Hall/CRC Res. Notes Math. 431, Chapman \& Hall/CRC, Boca Raton, 2002.

[LR 3] P. G. Lemarié-Rieusset, The Navier-Stokes equations in the critical Morrey-Campanato space, Rev. Mat. Iberoam. 23 (2007), 897-930.

[LR 4] P. G. Lemarié-Rieusset, Multipliers and Morrey spaces, Potential Anal. 38 (2013), 741752.

[LR 5] P. G. Lemarié-Rieusset, Erratum to "Multipliers and Morrey spaces", Potential Anal. 41 (2014), 1359-1362.

[LR 6] P. G. Lemarié-Rieusset, The Navier-Stokes Problem in the 21st Century, Chapman \& Hall/CRC, Boca Raton, 2016.

[Le] J. Leray, Essai sur le mouvement d'un liquide visqueux emplissant l'espace, Acta Math. 63 (1934), 193-248.

[Pr] G. Prodi, Un teorema di unicitá per le equazioni di Navier-Stokes, Ann. Mat. Pura Appl. 48 (1959), 173-182.

[Se] J. Serrin, The initial value problem for the Navier-Stokes equations, in: 1963 Nonlinear Problems (Proc. Sympos., Madison, 1962), Univ. of Wisconsin Press, Madison, Wis., 1963. 